Orthopäde 2019 · 48:1030-1035

https://doi.org/10.1007/s00132-019-03823-5

Online publiziert: 28. Oktober 2019

(C) Der/die Autor(en) 2019

\author{
M. Niedermeier' $\cdot$ A. Frühauf' · C. Bichler' $\cdot$ R. Rosenberger ${ }^{2} \cdot$ M. Kopp ${ }^{1}$ \\ ${ }^{1}$ Institut für Sportwissenschaft, Universität Innsbruck, Innsbruck, Österreich \\ ${ }^{2}$ Univ.-Klinik für Unfallchirurgie und Sporttraumatologie, Medizinische Universität Innsbruck, Innsbruck, \\ Österreich
}

\title{
Sport - zu Risiken und Nebenwirkungen
}

sogar als das größte Gesundheitsproblem unserer Zeit [43]. Die Weltgesundheitsorganisation empfiehlt daher für gesunde erwachsene Personen ein Mindestmaß von 150 min moderater körperlicher Aktivität pro Woche [48]. Das entspricht beispielsweise einem 30-minütigen Arbeitsweg, der 2- bis 3-mal wöchentlich zügig auf dem Fahrrad oder in flottem Tempo zu Fuß zurückgelegt wird. Neben präventiven Eigenschaften wird körperlicher Aktivität auch therapeutische Effektivität bei bereits bestehenden Erkrankungen zugesprochen [33]. „Exercise is medicine“, Bewegung als natürliches Medikament also, nennt sich eine globale Initiative des American College of Sports Medicine. Trotz Unterstützung dieser positiven Auswirkungen körperlicher Aktivität müssen in einer umfassenden Reflexion auch unerwünschte Wirkungen und Nebenwirkungen von Sport und Bewegung reflektiert werden.

Ziel dieses Beitrags ist es daher, potenzielle Risiken und Nebenwirkungen von Sport und körperlicher Aktivität zu beleuchten. Im Sinne des Biopsychosozialen Modells bilden biologische, psychologische und soziale Faktoren ein dynamisches Zusammenspiel in Entstehung und Verlauf von Gesundheitsstörungen [16]. Somit kann eine Abgrenzung zwischen psychischen und physischen $\mathrm{Ri}$ siken nicht klar definiert werden und das Vorhandensein von psychischen Risiken kann auch zu negativen körperlichen Auswirkungen und umgekehrt führen. Die folgende Einteilung in physische und psychische Risiken ist daher nicht als strikte Trennung zu sehen, sondern als Einteilung im Sinne der Übersichtlichkeit.

\section{Sport und physische Risiken}

\section{Verletzungen}

Physische Risiken der Sportausübungen spiegeln sich vor allem in Form von Verletzungen durch die Sportausübung wider. In der Europäischen Union können pro Jahr 6,1 Mio. Verletzungen ohne Todesfolge, die in einem Krankenhaus behandelt wurden, auf sportliche Betätigung zurückgeführt werden [5]. Das entspricht einer Inzidenz von Verletzungen ohne Todesfolge im Sport von etwa 1200 Verletzungen auf 100.000 Personen pro Jahr [5]. Im Vergleich zu Verletzungen in anderen Kategorien, wie Straßenverkehr (11\%), Haus und Freizeit (58\%) oder Arbeit (10\%) machen Sportverletzungen etwa $15 \%$ aller Verletzungen aus (• Tab. 1).

Insgesamt liegen Sportverletzungen also mit großem Abstand an zweiter Stelle hinter Verletzungen in Haus und Freizeit [5]. Sportartenspezifisch ereignen sich fast die Hälfte der Unfälle (40\%) in den Ballsportarten [20]; und hier im Besonderen im Fußball (70\% aller Ballsportunfälle) [5], wobei darauf hinzuweisen ist, dass die Daten nicht nach der Anzahl der sportausübenden Personen für die jeweiligen Sportarten adjustiert wurden. Die unteren Extremitäten sind bei Sportunfällen am häufigsten betroffen und vor allem Supinationstraumata mit Verletzungen des oberen Sprunggelenks gehören zu den häufigsten Verletzungen. Die Angaben zur Verletzungsinzidenz des oberen Sprunggelenks variieren je nach Sportart zwischen 0,06 (Kunstturnen) und 5 (Fußball) Verletzungen pro $1000 \mathrm{~h} \mathrm{Ex}$ - 
Tab. 1 Verletzungsstatistik der Mitgliedsländer der Europäischen Union der Jahre 2008-2010

Krankenhausfälle (stationär und ambulant)

\begin{tabular}{|c|c|c|}
\hline Haus und Freizeit & 22.865 .000 & 98.891 \\
\hline Sport & 6.063 .000 & 7000 \\
\hline Straßenverkehr & 4.192 .000 & 38.119 \\
\hline Arbeit/Schule & 4.629 .000 & 6211 \\
\hline
\end{tabular}

Daten beziehen sich auf ein Jahr. Adaptiert nach Bauer et al. 2014 [5] (Tab. 1, S. 676)

positionszeit [4]. Auch in der Schweiz steht Fußball an erster Stelle der Unfallstatistik [30]. An zweiter Stelle folgt das Skifahren auf Pisten und im Variantenbereich, wo zwar insgesamt weniger Verletzte, jedoch mehr Schwerverletzte und getötete Personen, verzeichnet wurden [30].

\section{》) Die Anzahl der Verletzungen mit Todesfolge bei der Sportausübung ist sehr gering}

Die Anzahl der Verletzungen mit Todesfolge bei der Sportausübung kann als sehr gering bezeichnet werden (• Tab. 1). Hier liegt die Kategorie Sport hinter den Kategorien Haus und Freizeit und Straßenverkehr. In der Schweiz machen Sportunfälle $5 \%$ der Todesfälle von Nichtberufsunfällen aus. Die meisten Todesfälle (80\%) geschehen im Haushalt und in der Freizeit und $14 \%$ im Straßenverkehr [30]. Für die Europäische Union werden zwischen 0,2 und 1,4 Verletzungen mit Todesfolge auf 100.000 Personen pro Jahr bei der Sportausübung genannt [5, 20]. Die Diskrepanz in den Zahlen ergibt sich vor allem durch die Inkludierung von Tod durch Ertrinken. Allerdings wurde in dieser Statistik nicht differenziert, ob jeder Tod durch Ertrinken während der Sportausübung geschehen ist, was zu einer erhöhten Zahl der tödlichen Sportunfälle geführt haben könnte. Bei den Verletzungen mit Todesfolge sind Schwimmen, Bergsport und Radfahren als die gefährlichsten Sportarten zu nennen [20]. Die Verletzungsrate mit Todesfolge beim Bergwandern aufgrund von Stürzen beträgt $6 \%$, wobei die meisten Stürze (75\%) beim Bergabgehen geschehen [17]. Dabei nicht inkludiert sind Todesfälle aufgrund kardiovaskulärer Ereignisse, die als Hauptursache für den tödlichen Ausgang von Bergwanderungen gelten [10].

Neben den genannten eher klassischen Sportarten erfahren in den letzten Jahren die sogenannten Hochrisikosportarten, wie zum Beispiel BaseJumping, Paragliding und Freeriding einen rasanten Zulauf an Ausübenden [32]. Unter Hochrisikosportarten werden in der Fachliteratur jene Sportarten diskutiert, bei denen eine erhöhte Wahrscheinlichkeit für eine schwere oder tödliche Verletzung direkt mit der Sportausübung assoziiert ist. Aufgrund des großen Zulaufs an Ausübenden wird angenommen, dass die Bedeutung der Hochrisikosportarten bei den Verletzungen steigen könnte, was sich auch in der aktuellen Forschung niederschlägt. Während frühere Forschung hauptsächlich auf Persönlichkeitsmerkmale fokussierte, gibt es mittlerweile einen Wandel in Richtung risikobewusste Verhaltensweisen, einschließlich dem Tragen von Schutzausrüstung, der Auswahl der Strecke und der Berücksichtigung von Wetterbedingungen [47]. Die Erhebung von relativen Unfallzahlen gestaltet sich bei Hochrisikosportarten als schwierig, da es keine genauen Zahlen zur Population unter Risiko gibt.

\section{Überlastungsschäden}

Wenn kein spezifisches, identifizierbares Ereignis (z.B. Trauma) für eine Verletzung ausgemacht werden kann, spricht man von Überlastungsschäden [11]. Überlastungsschäden machen einen großen Teil der Sportverletzungen aus (30-50\% der Sportverletzungen) [39]. Besonders in Sportarten, die lang andauernde Trainingseinheiten mit ähnlichem Bewegungsablauf beinhalten (und hier im Besonderen beim Laufen), aber auch in technischen Disziplinen mit einseitigen Bewegungen (Speerwurf, Golf,
Tennis) zeigen sich Überlastungsschäden $[11,14]$. Im Laufsport werden das mediale Schienbeinkantensyndrom und die Tendopathie der Achillessehne (je mit einer Prävalenz von bis zu $10 \%$ ) als häufige Überlastungsschäden angegeben [24]. Als Ursache für Überlastungsschäden generell werden vorangegangene Verletzungen, hoher Trainingsumfang, starke Anstiege in der Trainingsbelastung (Umfang und Intensität) sowie ungenügende Regeneration genannt [1, 14]. Speziell in der jugendlichen Wachstumsphase ist auf ein erhöhtes Risiko für Überlastungsschäden in Form von Gelenksschädigungen, Verletzungen der Epiphyse und Stressfrakturen zu verweisen [14].

Insbesondere bei der langjährigen Ausübung von Leistungssport können sich Überlastungsschäden in Form von Arthrosen auswirken, deren Lokalisation je nach Sportart unterschiedlich ist. Im Bereich der unteren Extremität besteht ein erhöhtes Risiko für Arthrosen im Knie- und Hüftgelenk in Kraftsportarten sowie in Sportarten mit schnellen Richtungswechseln [36]. Beispielsweise zeigt sich im Fußball, nicht jedoch im Laufsport, ein erhöhtes Risiko für Arthrose bei Elitesportlern im Vergleich zu Nichtelitesportlern [42]. Höheres Körpergewicht scheint mit einem erhöhten Risiko für Arthrosen im Knie- und Hüftgelenk assoziiert zu sein [36].

Im Bereich der Wirbelsäule finden sich degenerative Veränderungen bei Sportarten, in denen Hyperextensionsbelastungen mit Rotationsbelastungen verbunden werden. Dies trifft im Speziellen auf die Sportarten Turnen, Ringen, Leichtathletik (Hochsprung und einzelne Wurfdisziplinen) und Gewichtheben zu [36]. Hierbei zeigten sich auch Unterschiede in den betroffenen Segmenten der Wirbelsäule zwischen den Sportarten [36]. In der oberen Extremität finden sich nur wenige Untersuchungen zu Überlastungsschäden in Form von Arthrose. Es gibt aber Hinweise auf degenerative Gelenksveränderungen bei Speerwerfern und Golfern im Schulterbereich und bei den Sportarten Boxen und Klettern im Bereich der Hand [36]. 


\section{Plötzlicher Herztod}

Regelmäßige körperliche Aktivität hat in erster Linie eine protektive Wirkung auf das kardiovaskuläre System [40]. Nichtsdestotrotz können insbesondere intensive körperliche Belastungen bei kardiovaskulären Vorerkrankungen das Risiko für einen plötzlichen Herztod vorrübergehend erhöhen. Die Bestimmung der Prävalenz von plötzlichem Herztod während körperlicher Aktivität gestaltet sich als schwierig, da es ein sehr seltenes Ereignis ist und stark von Merkmalen der untersuchten Population (Alter, Vorerkrankungen, körperliche Aktivität) abhängt [41]. Bei sportlichen Aktivitäten in der Höhe, wie z. B. Bergwandern oder Skifahren, ist der plötzliche Herztod in der männlichen Population über 35 Jahre die häufigste Todesursache [10]. Bei sportlicher Aktivität im Allgemeinen zeigte sich bei einer Untersuchung von mehr als 20.000 Männer zwischen 40 und 84 Jahren über einen Zeitraum von 12 Jahren ein plötzlicher Herztodesfall auf 1,5 Mio. intensive körperliche Belastungen [2]. Schätzungsweise 6-17\% der plötzlichen Herztodesfälle sind auf intensive Belastung zurückzuführen [2]. Die Autoren merken an, dass die Prävalenz am höchsten bei körperlich inaktiven Personen ist, und empfehlen in Anbetracht der zusätzlichen positiven Auswirkungen eine regelmäßige körperliche Aktivität als die beste Vorsorge [2]. Etwa $70 \%$ der plötzlichen Herztodesfälle sind auf kardiale Vorerkrankungen zurückzuführen, wie postmortale Analysen gezeigt haben [37]. Daher wird im Falle des Vorliegens von kardiovaskulären oder metabolischen Risikofaktoren eine medizinische Untersuchung vor Beginn von Bewegungsprogrammen empfohlen [3].

\section{Beeinträchtigte Immunfunktion}

Ähnlich der Prävention von kardiovaskulären Erkrankungen kann durch regelmäßige körperliche Aktivität die Immunfunktion des Körpers positiv beeinflusst werden [38]. Vor allem bei längeren Bewegungseinheiten bis zur völligen Erschöpfung (z.B. Marathonlauf) kann die Immunfunktion des Körpers aber

Orthopäde 2019 - 48:1030-1035 https://doi.org/10.1007/s00132-019-03823-5

(c) Der/die Autor(en) 2019

\section{Niedermeier · A. Frühauf · C. Bichler · R. Rosenberger · M. Kopp} Sport - zu Risiken und Nebenwirkungen

\section{Zusammenfassung}

Sportliche Aktivität wird im Allgemeinen mit einer positiven Wirkung auf die Gesundheit in Verbindung gebracht. Unter verschiedenen Umständen kann Sportausübung aber riskant für die Gesundheit sein. Dies betrifft in erster Linie körperliche Beeinträchtigungen durch Verletzungen, die durch die Sportausübung entstehen können. Wenn Sport in einem exzessiven Umfang betrieben wird, erhöht sich zudem das Risiko für psychische Störungsbilder wie Essstörungen, Substanz- missbrauch sowie Sportabhängigkeit. Der vorliegende Beitrag hat zum Ziel, potenzielle Risiken und Nebenwirkungen, die mit Sportausübung verbunden sein können, zu beleuchten und in Relation zu den positiven Auswirkungen auf die Gesundheit zu setzen.

\section{Schlüsselwörter}

Abhängigkeitsverhalten · Essstörungen · Überlastungsschäden · Sportverletzungen . Substanzmissbrauch

\section{Exercise-risks and side effects}

Abstract

Physical activity and exercise is widely connected with positive effects on human health. However, exercise may also pose as a risk factor for health under specific circumstances. Primarily, the risks connected with exercise are physical risks, but also psychological risks may appear, especially when exercise is conducted excessively. Psychological risks include eating disorders, illegal and legal substance use and exercise dependence. The aims of the present article are to focus on potential risks and side effects of exercise and physical activity and to put the risks in the context of the positive effects of exercise on health.

\section{Keywords}

Addictive behavior - Eating disorders - Overuse injuries · Sport injuries · Substance abuse kurzfristig beeinträchtigt werden. Nach hochintensiven Trainingseinheiten ist die Immunfunktion zwischen 3 und $24 \mathrm{~h}$ beeinträchtigt [18]. Unter Marathonläufern zeigte sich, dass $13 \%$ innerhalb der Woche nach dem Marathon an einer Atemwegsinfektion erkrankten, während nur rund $2 \%$ der Personen, die sich zwar auf den Marathon vorbereitet hatten, letztendlich aber nicht teilnahmen, eine Atemwegsinfektion erlitten [29].

\section{Sport und psychische Risiken}

Vor allem exzessives Sporttreiben kann auch mit psychischen Risiken verbunden sein. Psychische Störungsbilder wie Depression oder Burnout werden am häufigsten bei jungen Athletinnen beschrieben, die Prävalenz unterscheidet sich jedoch kaum zwischen Sportlern und Nichtsportlern [46]. Bezogen auf den Breitensport gibt es Ausübungsmotive, die ungesunde psychische Faktoren begünstigen können. Im Gegensatz zur Sportmotivation aus gesundheitlichen Gründen haben Personen, die Sport treiben, um eine Verbesserung ihres äußeren Erscheinungsbildes zu erreichen, ein höheres Risiko für Körperunzufriedenheit, geringeren Selbstwert, geringeres Wohlbefinden und die Entwicklung eines gestörten Essverhaltens [44]. Dieser Aspekt könnte in der Zukunft aufgrund der intensivierten Nutzung sozialer Medien eine weitere Verschärfung erfahren.

\section{Essstörungen}

Athleten sind stärker als Nichtsportler gefährdet, an Essstörungen zu erkranken, wobei sich Essstörungen, wie Anorexia nervosa und Bulimia nervosa, nicht nur bei Athletinnen, sondern auch bei Athleten zeigen [45]. Menschen, die an Anorexia nervosa leiden, legen eine sehr niedrige Gewichtsschwelle für sich selbst fest und führen gezielt Gewichtsverluste her- 
bei, um diese zu erreichen. Betroffene leiden unter einer tiefsitzenden Angst, dick $\mathrm{zu}$ werden, wobei der Body Mass Index weniger als $17,5 \mathrm{~kg} / \mathrm{m}^{2}$ beträgt. Personen mit Bulimie zeigen abwechselnd Phasen mit erhöhter Nahrungszufuhr und der Kontrolle des Körpergewichts. Nach einer Essattacke wird mit unterschiedlichen Verhaltensweisen versucht, sich der kalorienreichen und als dickmachend bewerteten Nahrung wieder zu entledigen, beispielsweise durch selbst herbeigeführtes Erbrechen, Abführmittel, zeitweilige Hunger- und Fastenperioden, Einsatz von Appetitzüglern oder Sport.

\section{》) Je nach Sportart ist die Prävalenz von Essstörungen bei Athleten unterschiedlich}

Je nach Sportart ist die Prävalenz von Essstörungen bei Athleten unterschiedlich. Insbesondere in Sportarten, in denen durch Gewichtsreduktion ein Wettbewerbsvorteil erreicht werden kann (z.B. Ausdauersportarten wie Langstreckenlauf oder Sprungsportarten wie Skisprung), sowie in Sportarten mit Gewichtsklassen (z.B. Kampfsport) und ästhetischen Sportarten (z.B. Ballett oder Kunstturnen), zeigt sich eine erhöhte Prävalenz von Anorexia nervosa und Bulimia nervosa [12, 19]. Konkrete Prävalenzraten reichen von $2 \%$ in Ballsportarten bis zu über $40 \%$ in ästhetischen Sportarten wie Kunstturnen [19]. Bei diesen Sportarten wird empfohlen, das gesamte Betreuerteam auf Warnzeichen für Essstörungen zu schulen und die Bedeutung des Gewichts weniger stark in den Vordergrund $\mathrm{zu}$ rücken [19]. Bezüglich der Behandlung von Essstörungen bei Athleten wird darauf hingewiesen, dass eine Stigmatisierung der Betroffenen vermieden und proaktiv mit essstörungsassoziierten Problemen innerhalb des gesamten Teams umgegangen werden sollte [34]. Eine diagnostizierte Essstörung muss nicht zwangsläufig bedeuten, Athleten eine Teilnahme am Training und/oder an Wettkämpfen zu untersagen [34]. Bei einer Entscheidung, ob Athleten das Training fortführen oder an Wettkämpfen teilnehmen können, sollte allerdings das entscheidende Kriterium die Gesundheit der Athleten sein.

Mit der Essstörung gekoppelt treten häufig körperliche und psychische Folgeerkrankungen auf, die in weiterer Folge zu einem Verlust der Leistungsfähigkeit führen können [12]. Eine Besonderheit bei Athletinnen stellt dabei die Triade der sporttreibenden Frau dar, die neben einem gestörten Essverhalten Zyklusstörungen bis zum Ausbleiben der Periode und Osteoporose mit einhergehender Gefahr für Ermüdungsbrüche inkludiert [6]. Die Prävalenz des Ausbleibens der Periode in der weiblichen Allgemeinbevölkerung beträgt zwischen 2 und $5 \%$, verglichen mit bis zu $69 \%$ bei Sportarten, in denen Ästhetik und Gewicht eine leistungsrelevante Rolle spielt [27]. Hat sich die Triade bereits manifestiert, ist die Behandlung zur Wiederherstellung der Knochendichte oft langwierig oder gar nicht mehr möglich, weshalb der frühen Intervention bei gestörtem Essverhalten eine wichtige Bedeutung beigemessen wird [21].

Doch nicht nur weibliche, sondern auch männliche Leistungssportler können von einer Nährstoffunterversorgung betroffen sein. Das Modell zur Erfassung des relativen Energiedefizitsyndroms im Sport ist als Weiterentwicklung der Triade der sporttreibenden Frau zu betrachten, das Risikofaktoren für relative Energiedefizite von Leistungssportlern allgemein erfasst [26]. Hierbei wird das relative Energiedefizit als Ursache für weitere Beeinträchtigungen wie Störungen der Stoffwechselfunktionen, der Menstruation, der Knochengesundheit, des Immunsystems, der Proteinsynthese und der kardiovaskulären Gesundheit gesehen [26]. Psychische Beeinträchtigungen können dem Energiedefizit entweder vorangehen oder daraus resultieren.

\section{Sportabhängigkeit}

Einige Autoren sprechen unter bestimmten Voraussetzungen von einer Sportabhängigkeit [9]. Zu diesen Voraussetzungen zählen depressive Verstimmungen, wenn keine Sportausübung möglich ist, eine andauernde gedankliche Beschäftigung mit der Sportausübung, Sporttreiben trotz negativer gesundheitlicher
Hier steht eine Anzeige. 글 Springer 
Folgen oder entgegen ärztlichem Rat und die Vernachlässigung sozialer Kontakte, um mehr Sport betreiben zu können [9]. Empirische Daten in verschiedenen sportausübenden Populationen unterscheiden sich sehr stark und reichen, je nach Erhebungsmethode und Sportart, von 3-50\% [25]. Da nur ein geringer Prozentsatz der Bevölkerung überhaupt regelmäßig Sport betreibt, wird in der Gesamtbevölkerung von insgesamt geringen Prävalenzen ausgegangen (manifeste Störungsmerkmale: einer von 1000 Sportlern; behandlungsbedürftig: ca. einer von 10.000 Sportlern) [9]. Andere Autoren vermeiden eine Pathologisierung intensiver Sportausübung und sprechen von Sportleidenschaft auf einem Kontinuum zwischen stimmiger und zwanghafter Form [35]. Aus klinischer Sicht lässt sich bei eher zwanghafter Sportleidenschaft festhalten, dass speziell bei Läufern (auch im Hobbybereich) Stressfrakturen im Mittelfußbereich und bei Marathon- und Bergläufern zudem auch Überlastungsfrakturen im Fersenbein auftreten können. Typisch ist bei diesen Sportlern auch, dass das Training - gegen jede ärztliche Empfehlung nach Überlastungsschäden zu früh wieder forciert wird, wodurch es wieder zu einer Fraktur ebendort kommen kann. Entsprechend ist daher die Ergänzung einer "klassischen Frakturbehandlung“ durch eine "psychologische Trainingsbegleitung“ empfehlenswert.

\section{Substanzmissbrauch}

Die wohl wichtigste Assoziation im Bereich Sport und Substanzmissbrauch stellt die illegale Verwendung von leistungssteigernden Substanzen mit dem Ziel eines besseren Abschneidens bei sportlichen Wettkämpfen dar. Dabei besteht ein nicht $\mathrm{zu}$ unterschätzendes Gesundheitsrisiko für Sportler. Die Prävalenzraten reichen in verschiedenen Sportarten von 10-78\% [8, 15]. Die Wahrscheinlichkeit der Einnahme leistungssteigernder illegaler Substanzen steigt mit folgenden Charakteristika: Einnahme legaler Nahrungsergänzungsmittel, wahrgenommene soziale Normen im Sinne einer Dopingbefürwortung der
Umgebung und eine positive Einstellung zu Doping [31].

Substanzmissbrauch kann sich neben Doping auch auf Alkohol- und Drogenkonsum zum Zweck von Entspannung und Ablenkung beziehen. Allerdings zeigt sich keine eindeutige Tendenz, ob Sportausübung mit höherem oder niedrigem Alkoholkonsumverhalten assoziiert ist [23, 28]. Athleten scheinen aber im Vergleich zu ihrer Alterskohorte weniger Marihuana, Kokain oder psychedelische Substanzen zu konsumieren [13].

\section{Fazit für die Praxis}

- Sportliche Aktivitäten sind nicht gänzlich ohne Risiko. Die Gefahr von Verletzungen ist präsent, sollte jedoch in Relation zu anderen Risiken (z. B. Straßenverkehr) gesehen werden.

- Die Prophylaxe bezüglich negativer physischer Auswirkungen von Sport kann über verhaltensbezogene Zugänge gewährleistet werden, die von Präventivmaßnahmen zur direkten Verletzungsprophylaxe (z. B. Schutzkleidung) bis zu adäquater Regeneration zur Vermeidung von Überlastungsschäden reichen.

- Im Bereich der psychischen Risiken ist auf die Früherkennung und -intervention zu verweisen; es bedarf eines systemischen Zugangs, der sowohl Athleten, Betreuungspersonal als auch Funktionäre miteinschließt.

- Bei allen Risiken sollten die positiven gesundheitlichen Effekte von körperlicher Aktivität mitbeachtet werden. Es gibt umfassende Evidenz für zahlreiche gesundheitsfördernde Effekte körperlicher Aktivität - sowohl als Intervention als auch als Präventivmaßnahme gegen psychische und physische Gesundheitsstörungen.

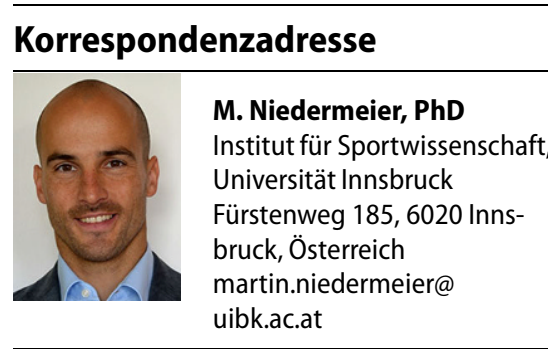

Funding. Open access funding provided by University of Innsbruck and Medical University of Innsbruck.

\section{Einhaltung ethischer Richtlinien}

Interessenkonflikt. M. Niedermeier, A. Frühauf, C. Bichler, R. Rosenberger und M. Kopp geben an, dass kein Interessenkonflikt besteht.

Für diesen Beitrag wurden von den Autoren keine Studien an Menschen oder Tieren durchgeführt. Für die aufgeführten Studien gelten die jeweils dort angegebenen ethischen Richtlinien.

Open Access Dieser Artikel wird unter der Creative Commons Namensnennung 4.0 International Lizenz (http://creativecommons.org/licenses/by/4.0/deed. de) veröffentlicht, welche die Nutzung, Vervielfältigung, Bearbeitung, Verbreitung und Wiedergabe in jeglichem Medium und Format erlaubt, sofern Sie den/die ursprünglichen Autor(en) und die Quelle ordnungsgemäß nennen, einen Linkzur Creative Commons Lizenz beifügen und angeben, ob Änderungen vorgenommen wurden.

\section{Literatur}

1. Aicale R, Tarantino D, Maffulli N (2018) Overuse injuries in sport: a comprehensive overview. JOrthop Surg Res 13:309

2. Albert CM, Mittleman MA, Chae CU et al (2000) Triggering of sudden death from cardiac causes by vigorous exertion. N Engl J Med 343:1355-1361

3. American College of Sports Medicine (2018) ACSM's guidelines for exercise testing and prescription. Lippincott, Williams, \& Wilkins, Philadelphia, PA

4. Bahr R, Engebretsen L (2011) Handbook of Sports Medicine and Science, Sports Injury Prevention. Wiley, New York, United States

5. Bauer R, Steiner M, Kisser R et al (2014) Unfälle in der EU. Bundesgesundheitsblatt Gesundheitsforschung Gesundheitsschutz 57:673-680

6. Birch K (2005) Female athlete triad. BMJ 330:244-246

7. Blair SN (2009) Physical inactivity: the biggest public health problem of the 21st century. $\mathrm{Br} J$ Sports Med 43:1-2

8. Blouin AG, Goldfield GS (1995) Body image and steroid use in male bodybuilders. Int J Eat Disord 18:159-165

9. Breuer S, Kleinert J (2009) Primäre Sportsucht und bewegungsbezogene Abhängigkeit - Beschreibung, Erklärung und Diagnostik. In: Batthyany D, Pritz A (Hrsg) Rausch ohne Drogen. Springer, Wien, S191-218

10. Burtscher M, Ponchia A (2010) The risk of cardiovascular events during leisure time activities at altitude. Prog Cardiovasc Dis 52:507-511 
11. Clarsen B,MyklebustG,BahrR(2013)Development and validation of a new method for the registration of overuse injuries in sports injury epidemiology: the Oslo Sports Trauma Research Centre (OSTRC) overuse injury questionnaire. Br J Sports Med 47:495-502

12. Currie A (2010) Sport and eating disorders understanding and managing the risks. Asian J Sports Med 1:34864

13. Diehl K, Thiel A, Zipfel S et al (2012) How healthy is the behavior of young athletes? A systematic literature review and meta-analyses. J Sports Sci Med 11:201-220

14. Difiori JP, Benjamin HJ, Brenner JS et al (2014) Overuse injuries and burnout in youth sports: a position statement from the American Medical Society for Sports Medicine. Br J Sports Med 48:287-288

15. Dunn M, Mazanov J, Sitharthan G (2009) Predicting future anabolic-androgenic steroid use intentions with current substance use: findings from an internet-based survey. Clin J Sport Med 19:222-227

16. Engel GL (1977) The need for a new medical model: a challenge for biomedicine. Science 196:129-136

17. Faulhaber M, Pocecco E, Niedermeier Met al (2017) Fall-related accidents among hikers in the Austrian Alps: a 9-year retrospective study. BMJ Open Sport ExercMed 3:e304

18. Gleeson M (2007) Immune function in sport and exercise. J Appl Physiol 103:693-699

19. Joy E, Kussman A, Nattiv A (2016) 2016 update on eating disorders in athletes: a comprehensive narrative review with a focus on clinical assessment and management. Br JSports Med 50:154-162

20. Kisser R, Bauer R (2012) The burden of sports injuries in the European Union. Research report D2h of the project "Safety in Sports". Austrian Road Safety Board (Kuratorium für Verkehrssicherheit), Vienna

21. Korsten-Reck U (2010) "Female athlete triad" und Stressfrakturen.J Gynäkol Endokrinol 8:230-235

22. Lee IM, Shiroma EJ, Lobelo F et al (2012) Effect of physical inactivity on major non-communicable diseases worldwide: an analysis of burden of disease and life expectancy. Lancet 380:219-229

23. Lisha NE, Sussman S, Fapa F et al (2013) Physical activity and alcohol use disorders. Am J Drug Alcohol Abuse 39:115-120

24. Lopes AD, Hespanhol LC, Yeung SS etal (2012) What are the main running-related musculoskeletal injuries? Sports Med 42:891-905

25. Magee CA, Buchanan I, Barrie L (2016) Profiles of exercise dependence symptoms in Ironman participants. Psychol Sport Exerc 24:48-55

26. Mountjoy $M$, Brackenridge $C$, Arrington $M$ et al (2016) International Olympic Committee consensus statement: harassment and abuse (non-accidental violence) in sport. Br J Sports Med 50:1019-1029

27. Nazem TG, Ackerman KE (2012) The female athlete triad. Sports Health 4:302-311

28. Niedermeier $M$, Frühauf $A$, Kopp-Wilfling $P$ et al (2018) Alcohol consumption and physical activity in Austrian college students-a cross-sectional study. Subst Use Misuse 53:1581-1590

29. Nieman DC, Johanssen LM, Lee JW et al (1990) Infectious episodes in runners before and after the Los Angeles Marathon. J Sports Med Phys Fitness 30:316-328

30. Niemann S, Lieb C, Sommer H (2015) Nichtberufsunfälle in derSchweiz: AktualisierteHochrechnung und Kostenberechnung. bfu - Beratungsstelle für Unfallverhütung, Bern, Schweiz
31. Ntoumanis N, Ng JY, Barkoukis V (2014) Personal and psychosocial predictors of doping use in physical activity settings: a meta-analysis. Sports Med 44:1603-1624

32. Pain MT, Pain MA (2005) Essay: Risk taking in sport. Lancet 1(366):33-34

33. Pedersen BK, Saltin B (2015) Exercise as medicine-evidence for prescribing exercise as therapy in 26 different chronic diseases. Scand J Med Sci Sports 3(25):1-72

34. Petrie TA, Greenleaf C (2013) Eating disorders. In: Papaioannou A, Hackfort D (Hrsg) Routledge companion to sport and exercise psychology, global perspectives and fundamental concepts. Routledge, London, United Kingdom, S 837-851

35. Robert JV, Jérémie V-F, Yvan P (2014) Passion for sport and exercise. In: Papaioannou A, Hackfort D (Hrsg) Routledge companion to sport and exercise psychology. Routledge, London, United Kingdom S668-683

36. Schmitt H (2006) Degenerative Gelenkerkrankungen nach Leistungssport. Dtsch Z Sportmed 57:248-254

37. Semsarian C, Ingles J, Wilde AA (2015) Sudden cardiac death in the young: the molecular autopsy and a practical approach to surviving relatives. Eur Heart J36:1290-1296

38. Simpson R, Kunz H, Agha N, Graff R (2015) Exercise and the regulation of immune functions. Prog $\mathrm{Mo}$ Biol Transl Sci 135:355-380. https://doi.org/10. 1016/bs.pmbts.2015.08.001

39. Sobhani S, Dekker R, Postema K et al (2013) Epidemiology of ankle and foot overuse injuries in sports: a systematic review. Scand J Med Sci Sports 23:669-686

40. Thompson PD (2003) Exercise and physical activity in the prevention and treatment of atherosclerotic cardiovascular disease: a statement from the Council on Clinical Cardiology (Subcommittee on Exercise, Rehabilitation, and Prevention) and the Council on Nutrition, Physical Activity, and Metabolism (Subcommittee on Physical Activity). Arterioscler Thromb Vasc Biol 23:42-49

41. Thompson PD, Franklin BA, Balady GJ et al (2007) Exercise and acute cardiovascular events placing the risks into perspective: a scientific statement from the American Heart Association Council on Nutrition, Physical Activity, and Metabolism and the Council on Clinical Cardiology. Circulation 115:2358-2368

42. Tran G, Smith TO, Grice A et al (2016) Does sports participation (including level of performance and previous injury) increase risk of osteoarthritis? A systematic review and meta-analysis. Br J Sports Med 50:1459-1466

43. Trost SG, Blair SN, Khan KM (2014) Physical inactivity remains the greatest public health problem of the 21 st century: evidence, improved methods and solutions using the " 7 investments that work" as a framework. Br J Sports Med 48:169-170

44. Vartanian LR, Wharton CM, Green EB (2012) Appearance vs. health motives for exercise and for weight loss. Psychol Sport Exerc 13:251-256

45. Werner A, Thiel A, Schneider S et al (2013) Weightcontrol behaviour and weight-concerns in young elite athletes-a systematic review. J Eat Disord 1:18-18

46. Wolanin A, Hong E, Marks D et al (2016) Prevalence of clinically elevated depressive symptoms in college athletes and differences by gender and sport. Br J Sports Med 50:167-171

47. Woodman T, Hardy L, Barlow Met al (2010) Motives for participation in prolonged engagement high-risk sports: an agentic emotion regulation perspective. Psychol Sport Exerc 11:345-352

48. World Health Organization (2010) Global recommendations on physical activity for health. World Health Organization, Switzerland, Geneva 